\title{
SUPPLEMENTAL DNA SEQUENCES
}

\section{Sequences of synthetic $m 8$ and $S u(H)$ UAS constructs}

DNA sequence is followed by protein sequence for each construct. For DNA; EcoRI and XbaI cloning sites underlined, start-ATG underlined-bold and mutated bases green. For protein; FLAG-tag blue-bold; HA-tag purple, bold; spacer red-bold; mutated "Ck2" amino acids green; mutated "deg” amino acids magenta; and Vp16 domain yellow.

\section{m8-FLAG}

gaattcaaccaaaATGGCCGACTACAAGGACGACGACGACAAGGGCCCCCCCGGCATGGAGTACA CCACCAAGACCCAGATCTACCAGAAGGTGAAGAAGCCCATGCTGGAGCGCCAGCGCCGCG CCCGCATGAACAAGTGCCTGGACAACCTGAAGACCCTGGTGGCCGAGCTGCGCGGCGACGA CGGCATCCTGCGCATGGACAAGGCCGAGATGCTGGAGTCCGCCGTGATCTTCATGCGCCAG CAGAAGACCCCCAAGAAGGTGGCCCAGGAGGAGCAGTCCCTGCCCCTGGACTCCTTCAAGA ACGGCTACATGAACGCCGTGAACGAGGTGTCCCGCGTGATGGCCTCCACCCCCGGCATGTC CGTGGACCTGGGCAAGTCCGTGATGACCCACCTGGGCCGCGTGTACAAGAACCTGCAGCAG TTCCACGAGGCCCAGTCCGCCGCCGACTTCATCCAGAACTCCATGGACTGCTCCTCCATGGA CAAGGCCCCCCTGTCCCCCGCCTCCTCCGGCTACCACTCCGACTGCGACTCCCCCGCCCCCT CCCCCCAGCCCATGCAGCAGCCCCTGTGGCGCCCCTGGtgataatagtctaga

MADYKDDDDKGPPGMEYTTKTQIYQKVKKPMLERQRRARMNKCLDNLKTLVAELRG DDGILRMDKAEMLESAVIFMRQQKTPKKVAQEEQSLPLDSFKNGYMNAVNEVSRVMAS TPGMSVDLGKSVMTHLGRVYKNLQQFHEAQSAADFIQNSMDCSSMDKAPLSPASSGYH SDCDSPAPSPQPMQQPLWRPW

\section{$\underline{\mathrm{m} 8^{\mathrm{Ck} 2} \text {-FLAG }}$}

gaattcaaccaaaATGGCCGACTACAAGGACGACGACGACAAGGGCCCCCCCGGCATGGAG TACACCACCAAGACCCAGATCTACCAGAAGGTGAAGAAGCCCATGCTGGAGCGCCA GCGCCGCGCCCGCATGAACAAGTGCCTGGACAACCTGAAGACCCTGGTGGCCGAGC TGCGCGGCGACGACGGCATCCTGCGCATGGACAAGGCCGAGATGCTGGAGTCCGCC GTGATCTTCATGCGCCAGCAGAAGACCCCCAAGAAGGTGGCCCAGGAGGAGCAGTC CCTGCCCCTGGACTCCTTCAAGAACGGCTACATGAACGCCGTGAACGAGGTGTCCCG CGTGATGGCCTCCACCCCCGGCATGTCCGTGGACCTGGGCAAGTCCGTGATGACCCA CCTGGGCCGCGTGTACAAGAACCTGCAGCAGTTCCACGAGGCCCAGTCCGCCGCCGA 
CTTCATCCAGAACTCCATGGACTGCTCCTCCATGGACAAGGCCCCCCTGgCCCCCGCC TCCgCCGGCTACCACgCCGACTGCGACTCCCCCGCCCCCTCCCCCCAGCCCATGCAGC AGCCCCTGTGGCGCCCCTGGtgataatagtctaga

MADYKDDDDKGPPGMEYTTKTQIYQKVKKPMLERQRRARMNKCLDNLKTLVAELRG DDGILRMDKAEMLESAVIFMRQQKTPKKVAQEEQSLPLDSFKNGYMNAVNEVSRVMAS TPGMSVDLGKSVMTHLGRVYKNLQQFHEAQSAADFIQNSMDCSSMDKAPLAPASAGY HADCDSPAPSPQPMQQPLWRPW

\section{$\underline{\text { Su(H)-FLAG-HA }}$}

gaattcaaccaaaATGGCCGACTACAAGGACGACGACGACAAGGGCCCCCCCGGCATGAAGTCCT ACTCCCAGTTCAACCTGAACGCCGCCGCCCCCCCCGCCATCGCCTACGAGACCACCGTGGT GAACCCCAACGGCTCCCCCCTGGACCCCCACCAGCAGCAGCAGCAGCAGTCCCAGGACATG CCCCACTTCGGCCTGCCCGGCCCCCAGCCCCССTCCTCCCAGCAGCAGCAGCAGCAGCTGC AGGTGCACCACCAGCAGCAGCAGCAGCAGCAGCAGCAGCAGCAGCAGCAGCAGCACCAGC AGCAGATGCAGATGTCCCTGCTGCCCGGCCCCTACCGCCCCCACATCGAGGAGAAGAAGCT GACCCGCGACGCCATGGAGAAGTACATGCGCGAGCGCAACGACATGGTGATCGTGATCCTG CACGCCAAGGTGGCCCAGAAGTCCTACGGCAACGAGAAGCGCTTCTTCTGCCCCCCСCCCT GCATCTACCTGTTCGGCTCCGGCTGGCGCCGCCGCTACGAGGAGATGCTGCAGCAGGGCGA GGGCGAGCAGGGCGCCCAGCTGTGCGCCTTCATCGGCATCGGCTCCTCCGACCAGGACATG CAGCAGCTGGACCTGAACGGCAAGCAGTACTGCGCCGCCAAGACCCTGTTCATCTCCGACT CCGACAAGCGCAAGCACTTCATGCTGTCCGTGAAGATGTTCTACGGCAACGGCCACGACAT CGGCGTGTTCAACTCCAAGCGCATCAAGGTGATCTCCAAGCCCTCCAAGAAGAAGCAGTCC CTGAAGAACGCCGACCTGTGCATCGCCTCCGGCACCAACGTGGCCCTGTTCAACCGCCTGC GCTCCCAGACCGTGTCCACCCGCTACCTGCACGTGGAGAACGGCCACTTCCACGCCTCCTCC ACCCAGTGGGGCGCCTTCACCATCCACCTGCTGGACGACAACGAGTCCGAGTCCGAGGAGT TCCAGGTGCGCGACGGCTACATCCACTACGGCGCCACCGTGAAGCTGGTGTGCTCCGTGAC CGGCATGGCCCTGCCCCGCCTGATCATCCGCAAGGTGGACAAGCAGATGGCCCTGCTGGAG GCCGACGACCCCGTGTCCCAGCTGCACAAGTGCGCCTTCTACATGAAGGACACCGACCGCA TGTACCTGTGCCTGTCCCAGGAGAAGATCATCCAGTTCCAGGCCACCCCCTGCCCCAAGGA GCCCAACAAGGAGATGATCAACGACGGCGCCTGCTGGACCATCATCTCCACCGACAAGGCC GAGTACCAGTTCTACGAGGGCATGGGCCCCGTGGCCTCCCCCGTGACCCCCGTGCCCATCGT GAACTCCCTGAACCTGAACGGCGGCGGCGACGTGGCCATGCTGGAGCTGTCCGGCGACAAC TTCACCCCCCACCTGCAGGTGTGGTTCGGCGACGTGGAGGCCGAGACCATGTACCGCTGCA CCGAGACCCTGCTGTGCGTGGTGCCCGAGATCTCCCAGTTCCGCGGCGAGTGGCTGTGGGT GCGCCAGCCCACCCAGGTGCCCATCTCCCTGGTGCGCAACGACGGCATCATCTACGCCACC GGCCTGACCTTCACCTACACCCCCGAGCCCGGCCCCCGCCCCCACTGCAACACCCAGGCCG AGGACGTGATGCGCGCCCGCCAGAACAACAACAACAACAACATCACCTCCATCTCCAACAA CAACAACTCCAACAACGCCGGCTCCCCCGCCGCCGGCGGCGGCCTGCAGCAGCAGCAGCAG 
CAGCACCAGGCCCTGCCСTCCATCTCCGAGGTGCAGTGGAACTCCCACGGCTCCGGCCTGTC CGGCCCCCCCGGCTACCCCTACGACGTGCCCGACTACGCCtgataatagtctaga

MADYKDDDDKGPPGMKSYSQFNLNAAAPPAIAYETTVVNPNGSPLDPHQQQQQQSQD MPHFGLPGPQPPSSQQQQQQLQVHHQQQQQQQQQQQQQQHQQQMQMSLLPGPYRPHI EEKKLTRDAMEKYMRERNDMVIVILHAKVAQKSYGNEKRFFCPPPCIYLFGSGWRRRY EEMLQQGEGEQGAQLCAFIGIGSSDQDMQQLDLNGKQYCAAKTLFISDSDKRKHFMLS VKMFYGNGHDIGVFNSKRIKVISKPSKKKQSLKNADLCIASGTNVALFNRLRSQTVSTRY LHVENGHFHASSTQWGAFTIHLLDDNESESEEFQVRDGYIHYGATVKLVCSVTGMALPR LIIRKVDKQMALLEADDPVSQLHKCAFYMKDTDRMYLCLSQEKIIQFQATPCPKEPNKE MINDGACWTIISTDKAEYQFYEGMGPVASPVTPVPIVNSLNLNGGGDVAMLELSGDNFT PHLQVWFGDVEAETMYRCTETLLCVVPEISQFRGEWLWVRQPTQVPISLVRNDGIIYAT GLTFTYTPEPGPRPHCNTQAEDVMRARQNNNNNNITSISNNNNSNNAGSPAAGGGLQQQ QQQHQALPSISEVQWNSHGSGLSGPPGYPYDVPDYA 